\title{
ON INTERVAL ESTIMATION FOR THE NUMBER OF SIGNALS
}

\author{
Pinyuen Chen \\ Department of Mathematics, Syracuse University \\ 215 Carnegie, Syracuse, NY 13244, USA \\ phone: + (01) 315-443-1577, fax: + (01) 315-443-1475, email: pinchen@syr.edu
}

\begin{abstract}
We propose a multi-step procedure for constructing a confidence interval for the number of signals present. The proposed procedure uses the ratios of a sample eigenvalue and the sum of different sample eigenvalues sequentially to determine the upper and lower limits for the confidence interval. A preference zone in the parameter space of the population eigenvalues is defined to separate the signals and the noise. We derive the probability of a correct estimation, $P(C E)$, and the least favorable configuration (LFC) asymptotically under the preference zone. Some important procedure properties are shown. Under the asymptotic LFC, the $P(C E)$ attains its minimum over the preference zone in the parameter space of all eigenvalues. Therefore a minimum sample size can be determined in order to implement our procedure with a guaranteed probability requirement.
\end{abstract}

\section{INTRODUCTION}

In this paper, we propose a multi-step procedure for constructing a confidence interval for the number of signals. The procedure combines the results of two multi-step estimations. The two estimations provide the upper limit and the lower limit respectively for the confidence interval. A framework adopted from the selection theory is used to set up the problem and then a selection-type procedure is proposed to solve the problem. Schmidt [1], Wax and Kailath [2], Wax, Shan, and Kailath [3], and Zhao, Krishnaiah, and Bai [4] modeled the signal and noise in certain manner so that the number of signals present in radar measurements can be considered as the difference of the components in an observed vector and the multiplicity of the common smallest eigenvalue of the covariance matrix. Chen [5] developed a single step procedure for estimating the multiplicity of the smallest eigenvalue and applied their procedure to a measured radar data. The procedure in [5] is a single-step procedure and it provides an upper confidence limit for the number of signals with a guaranteed probability requirement. This paper considers a multi-step procedure to construct a confidence interval for the number of signals with a guaranteed confidence.

Analogous to the approach by Chen [5], our proposed procedure is developed under the framework of statistical ranking and selection theory. The literature on ranking and selection theory is dominated by two methods; the indifference zone and the subset selection formulations. Chen, Melvin, and Wicks [6] used a variation of the subset selection approach for developing a screening procedure. Their results showed dramatically improved performance over conventional techniques. Formulations and procedures for selection from a single multivariate normal population was thoroughly reviewed in Gupta and Panchapakesan [7], Chapters 7 and 14 and more recently in Gupta and Panchapakesan [8], Section 2.

The formulation that is the closest to this paper is the approach in Chen and Wicks [9], where a multi-step selection procedure was developed to estimate the lower confidence limit of the number of signal present. This paper generalizes their result of a lower confidence limit to a confidence interval by using a combination of two multistep procedure. The first part consists of a step up procedure for the lower confidence limit and the second part consists of a step down procedure for the upper confidence limit.

In Section 2 below, we introduce our proposed procedure and derive the least favourable configuration of the procedure. In addition, we state and prove properties of the proposed procedure in Section 2. In Section 3, we give illustrative examples.

\section{PROPROSED PROCEDURE}

In this section, we propose a step-up procedure for constructing an upper confidence limit for the number of signals present and a step-down procedure for constructing a lower limit for the number of signals present. The combining result of the two procedures gives a confidence interval for the number of signals present. We also study the properties of the procedure. The procedure is based on the ratios of the eigenvalues of the sample covariance matrix from a multivariate normal data. First we give a description of the underlying model to our problem.

As described in Schmidt [1], Wax and Kailath [2], Wax, Shan, and Kailath [3], and Zhao, Krishnaiah, and Bai [4], the measured data vector in radar signal processing, denoted by the $\mathrm{p} \times 1$ vector $\boldsymbol{x}(\mathrm{t})$, can be written as 
$\mathrm{x}(\mathrm{t})=\sum_{1}^{\mathrm{q}} \mathrm{A}\left(\Phi_{\mathrm{i}}\right) \mathrm{s}_{\mathrm{i}}(\mathrm{t})+\mathrm{n}(\mathrm{t})=\operatorname{As}(\mathrm{t})+\mathrm{n}(\mathrm{t})$

where

$\mathrm{A}=\left[\mathrm{A}\left(\Phi_{1}\right), \ldots, \mathrm{A}\left(\Phi_{\mathrm{q}}\right)\right], \mathrm{s}(\mathrm{t})=\left(\mathrm{s}_{1}(\mathrm{t}) \ldots \mathrm{s}_{\mathrm{q}}(\mathrm{t})\right)^{\prime}$,

$\mathrm{n}(\mathrm{t})=\left(\mathrm{n}_{1}(\mathrm{t}), \ldots, \mathrm{n}_{\mathrm{p}}(\mathrm{t})\right)^{\prime}$

and $\mathrm{q}<\mathrm{p}$. The extreme case where $\mathrm{q}=\mathrm{p}$ represents an unrealistic situation, as can be seen in equation (1) of [2]. Therefore it is not addressed in this paper. In the above model, $\boldsymbol{n}(\mathrm{t})$ is a $\mathrm{p} \times 1$ complex vector referred to as additive noise. It is distributed as complex multivariate normal with mean vector 0 and covariance matrix $\sigma^{2} I_{p}$ where $\sigma^{2}$ is unknown and $I_{p}$ is $\mathrm{p} \times \mathrm{p}$ identity matrix and $s_{\mathrm{i}}(\mathrm{t})$ is a scalar complex waveform associated with the ith signal $\boldsymbol{s}(\mathrm{t})$ is distributed as complex multivariate normal with mean vector 0 and nonsingular covariance matrix $\psi$. Note that $\boldsymbol{n}(\mathrm{t})$ is independent of $\boldsymbol{s}(\mathrm{t}) . \mathrm{A}\left(\Phi_{\mathrm{i}}\right)$ is a $\mathrm{p} \times 1$ complex vector, described by an unknown vector parameter $\Phi_{i}$ associated with the ith signal. We are interested in determining the number of signals $\mathrm{q}$ from a sample $x\left(t_{1}\right), x\left(t_{2}\right), \ldots, x\left(t_{n}\right)$ using statistical ranking and selection theory.

The covariance matrix $\Sigma$ of $\boldsymbol{x}(\mathrm{t})$ is given by

$$
\Sigma=\mathrm{A} \Psi \overline{\mathrm{A}^{\prime}}+\sigma^{2} \mathrm{I}_{\mathrm{p}}
$$

where $\overline{\mathrm{A}^{\prime}}$ denotes the conjugate transpose of $\mathrm{A}$. Let $\lambda_{1} \geq \lambda_{2} \geq \ldots \geq \lambda_{p}$ denote the positive eigenvalues of the covariance matrix $\Sigma$ and define the hypothesis, for $\mathrm{q}<\mathrm{p}$, $\mathrm{H}_{\mathrm{q}}: \lambda_{\mathrm{i}}=\sigma^{2}+\theta_{\mathrm{i}}(\mathrm{i}=1,2, \ldots, \mathrm{q})$;

$\lambda_{q+j}=\sigma^{2}(j=1,2, \ldots, p-q)$,

where $\theta_{i}$ 's are the $q(<p)$ positive eigenvalues of $\mathrm{A} \Psi \overline{\mathrm{A}^{\prime}}$.

Therefore $\mathrm{H}_{\mathrm{q}}$ is equivalent to the hypothesis that $\mathrm{q}$ signals are transmitted. [2] used Akaike's Information Criterion (AIC) and Schwartz Rissanen's Minimum Description Length (MLD) criterion for model selection to determine the value q. [4] also used an information theoretic criterion to estimate $\mathrm{q}$.

The following Definition 1 was considered by [2] and [4]. It is also required for our model.

\section{Definition 1:}

$$
\begin{gathered}
H_{q}: \lambda_{i}=\sigma^{2}+\theta_{i}(i=1,2, \ldots, q) ; \lambda_{q+j}=\sigma^{2} \\
\quad(j=1,2, \ldots, p-q) .
\end{gathered}
$$

That is, the multiplicity of the smallest eigenvalue is $\mathrm{p}-\mathrm{q}$, where $\mathrm{p}$ is known and $\mathrm{q}$ is unknown. Moreover, we assume that $\theta_{1}>\theta_{2}>\ldots>\theta_{\mathrm{q}}$. This is a reasonable assump- tion because $\theta_{1}, \theta_{2}, \ldots, \theta_{\mathrm{q}}$ came from the first term of the covariance matrix in equation (2.2).

The least favorable configuration is the parameter configuration where the probability of a correct separation is minimized. In order to find the minimum sample size to achieve certain probability requirement, we need to derive the least favorable configuration under a specific zone. To do that, we require the following definition.

Definition 2: When $\mathrm{q}>1$,

$$
\left.\Omega_{\mathrm{S}}=\left\{\left(\lambda_{1}, \ldots, \lambda_{\mathrm{p}}\right) \mid \lambda_{\mathrm{q}} / \lambda_{\mathrm{p}}\right) \geq \delta^{*}, \lambda_{\mathrm{q}+1} / \lambda_{\mathrm{p}}=1\right\},
$$

a subspace in the parameter space containing all the parameters $\left(\lambda_{1}, \ldots, \lambda_{p}\right)$, is called the preference zone in ranking and selection theory. The case $\mathrm{q}=0$ corresponds to the case where there is no signal. The only configuration for the parameter when $\mathrm{q}=0$ is the equal parameter configuration, i.e., $\lambda_{1} / \lambda_{p}=1$. It is in the preference zone, we seek for the least favorable configuration where the probability of a correct estimation is minimized.

We define two disjoint and exhaustive subsets $\Omega_{\mathrm{S}}$ and $\Omega_{\mathrm{N}}$ of $\Omega=\left\{\left(\lambda_{1}, \ldots, \lambda_{\mathrm{p}}\right) \mid \lambda_{1} \geq \ldots \geq \lambda_{\mathrm{p}}>0\right\}$ by using ratio as the "distance measure". The set $\Omega_{\mathrm{S}}$ consists of the eigenvalues "sufficiently" away from the smallest eigenvalue $\lambda_{\mathrm{p}}$, and the set $\Omega_{\mathrm{N}}$ is the complement of $\Omega_{\mathrm{S}}$. In other words, we define

$$
\begin{gathered}
\mathrm{d}\left(\lambda_{\mathrm{i}}, \lambda_{\mathrm{j}}\right)=\lambda_{\mathrm{i}} / \lambda_{\mathrm{j}} ; \\
\Omega_{\mathrm{S}}=\left\{\lambda_{\mathrm{i}}, \mathrm{i}=1,2,3, \ldots, \mathrm{p}-1 \mid \mathrm{d}\left(\lambda_{\mathrm{i}}, \lambda_{\mathrm{p}}\right) \geq \delta^{*}\right\}
\end{gathered}
$$

and $\Omega_{\mathrm{N}}=\Omega-\Omega_{\mathrm{S}}$, where $\delta^{*}>1$ is a pre-assigned real number used to differentiate among eigenvalues (signal and noise). Our goal is to construct a $100\left(1-\mathrm{P}^{*}\right) \%$ confidence interval for the unknown cardinality of $\Omega_{\mathrm{S}}$, q, the number of signals. That is, we need to construct a pair of statistics such that $\mathrm{P}(\mathrm{q} \in[\underline{\mathrm{q}}, \overline{\mathrm{q}}]) \geq \mathrm{P}^{*}$. The process of finding the lower and upper limits $\{\underline{q}, \overline{\mathrm{q}}\}$ is defined as follows.

Part I: A step-up procedure $\underline{\mathrm{R}}$ for finding the lower limit $\underline{\mathrm{q}}$.

In this part, we require a procedure $\underline{\mathrm{R}}$ to separate the set of eigenvalues into two disjoint subsets, $\underline{S_{s}}$ and $\underline{S_{n}}$. The separation is said to be correct ( $\underline{\mathrm{CE}}$ ) if $\mathrm{S}_{\mathrm{S}} \subseteq \Omega_{\mathrm{S}}$, meaning that the true values of all the eigenvalues in the selected subset are significantly larger than the true smallest eigenvalue. In other words, $\underline{\mathrm{CE}}$ occurs when the value of $\mathrm{q}$ is larger than or equal to the number of elements in $\mathrm{S}_{\mathrm{S}}$. The procedure $\underline{\mathrm{R}}$ should satisfy the predetermined prob- 
ability requirement that $\mathrm{P}(\underline{\mathrm{CE}} \mid \underline{\mathrm{R}}) \geq\left(1+\mathrm{P}^{*}\right) / 2$, where $\mathrm{P}^{*}$, satisfying $1 / \mathrm{p}<\left(1+\mathrm{P}^{*}\right) / 2<1$, is specified in advance.

Procedure $\underline{\mathrm{R}}$ : Compute the covariance matrices $\mathrm{S}$. Let $\ell_{1}>\ell_{2}>\ldots>\ell_{\mathrm{p}}$ denote the ordered eigenvalues of $\mathrm{S}$. Let $\underline{\mathrm{c}}_{\mathrm{i}}=\max \left(\delta^{*}, \underline{\mathrm{p}}_{\mathrm{i}}\right)$ where $\underline{\mathrm{p}}_{\mathrm{i}}$ denotes the $100\left(1+\mathrm{P}^{*}\right) / 2$ percentile of the random variable $\ell_{\mathrm{i}} /\left[\left(\ell_{\mathrm{p}}+\ell_{\mathrm{p}-1}+\ldots+\ell_{\mathrm{i}+1}\right) /(\mathrm{p}-\mathrm{i})\right]$ when the true number of signals is $q=i-1(i=1, \ldots, p-1)$, and the $p$ eigenvalues of the matrix $\Sigma$ satisfy the slippage configuration condition:

$$
\lambda_{1}=\lambda_{2}=\ldots=\lambda_{\mathrm{i}-1}>\lambda_{\mathrm{i}}=\ldots=\lambda_{\mathrm{p}}, \lambda_{\mathrm{i}-1} / \lambda_{\mathrm{i}}=\delta^{*}>1
$$

Let $\mathrm{q}$ denote the $100\left(1+\mathrm{P}^{*}\right) / 2$ lower confidence limit of $\mathrm{q}$. Claim that $\underline{\mathrm{q}}=0$

if $\ell_{1} /\left[\left(\ell_{\mathrm{p}}+\ell_{\mathrm{p}-1}+\ldots+\ell_{2}\right) /(\mathrm{p}-1)\right]<\underline{\mathrm{c}}_{1}$. Otherwise, go to next step.

Claim that $\underline{\mathrm{q}}=1$

if $\ell_{1} /\left[\left(\ell_{\mathrm{p}}+\ell_{\mathrm{p}-1}+\ldots+\ell_{2}\right) /(\mathrm{p}-1)\right]>\underline{\mathrm{c}}_{1}$

and $\ell_{2} /\left[\left(\ell_{\mathrm{p}}+\ell_{\mathrm{p}-1}+\ldots+\ell_{3}\right) /(\mathrm{p}-2)\right]<\underline{\mathrm{c}}_{2}$. Otherwise, go to next step.

Claim that $\underline{\mathrm{q}}=\mathrm{i}$ if

$\ell_{1} /\left[\left(\ell_{\mathrm{p}}+\ell_{\mathrm{p}-1}+\ldots+\ell_{2}\right) /(\mathrm{p}-1)\right]>\underline{\mathrm{c}}_{1}, \ldots$,

$\ell_{\mathrm{i}} /\left[\left(\ell_{\mathrm{p}}+\ell_{\mathrm{p}-1}+\ldots+\ell_{\mathrm{i}+1}\right) /(\mathrm{p}-\mathrm{i})\right]>\underline{\mathrm{c}}_{\mathrm{i}}$, and

$\ell_{\mathrm{i}+1} /\left[\left(\ell_{\mathrm{p}}+\ell_{\mathrm{p}-1}+\ldots+\ell_{\mathrm{i}+2}\right) /(\mathrm{p}-\mathrm{i}-1)\right]<\underline{\mathrm{c}}_{\mathrm{i}+1}$. Otherwise, go to next step.

Claim that $\underline{\mathrm{q}}=\mathrm{p}-1$ if

$\ell_{1} /\left[\left(\ell_{\mathrm{p}}+\ell_{\mathrm{p}-1}+\ldots+\ell_{2}\right) /(\mathrm{p}-1)\right]>\underline{\mathrm{c}}_{1}, \ldots$,

and $\ell_{\mathrm{p}-1} / \ell_{\mathrm{p}}>\underline{\mathrm{c}}_{\mathrm{p}-1}$.

Part II: A step-down procedure $\overline{\mathrm{R}}$ for finding the upper $\operatorname{limit} \overline{\mathrm{q}}$.

In this part, we require a procedure $\overline{\mathrm{R}}$ to separate the set of eigenvalues into two disjoint subsets, $\overline{\mathrm{S}_{\mathrm{s}}}$ and $\overline{\mathrm{S}_{\mathrm{n}}}$. The separation is said to be correct $(\overline{\mathrm{CE}})$ if $\overline{\mathrm{S}_{\mathrm{S}}} \supseteq \Omega_{\mathrm{S}}$, meaning that all the eigenvalues whose true values are significantly larger than the smallest eigenvalue are included in the selected subset. In other words, $\overline{\mathrm{CE}}$ occurs when the value of $\mathrm{q}$ is smaller than or equal to the number of elements in $\mathrm{S}_{\mathrm{S}}$. The procedure $\overline{\mathrm{R}}$ should satisfy the predetermined probability requirement that $\mathrm{P}(\overline{\mathrm{CE}} \mid \overline{\mathrm{R}}) \geq$ $\left(1+\mathrm{P}^{*}\right) / 2$, where $\mathrm{P}^{*}$, satisfying $1 / \mathrm{p}<\left(1+\mathrm{P}^{*}\right) / 2<1$, is specified in advance.

Procedure $\overline{\mathrm{R}}$ : Let $\overline{\mathrm{c}}_{\mathrm{i}}=\min \left(\delta^{*}, \overline{\mathrm{p}}_{\mathrm{i}}\right)$ where $\overline{\mathrm{p}}_{\mathrm{i}}$ denotes the $100\left(1-\mathrm{P}^{*}\right) / 2$ percentile of the random variable $\ell_{\mathrm{i}} /\left[\left(\ell_{\mathrm{p}}+\ell_{\mathrm{p}-1}+\ldots+\ell_{\mathrm{i}+1}\right) /(\mathrm{p}-\mathrm{i})\right]$ when the true number of signals is $q=i \quad(i=1, \ldots, p-1)$, and the $p$ eigenvalues of the matrix $\Sigma$ satisfy the slippage configuration condition as defined in (2.5). Let $\overline{\mathrm{q}}$ denote the $100\left(1+\mathrm{P}^{*}\right) / 2$ upper confidence limit of $q$.

Claim that $\overline{\mathrm{q}}=\mathrm{p}-1$

if $\ell_{\mathrm{p}-1} / \ell_{\mathrm{p}}>\overline{\mathrm{c}}_{\mathrm{p}-1}$. Otherwise, go to next step.

Claim that $\overline{\mathrm{q}}=\mathrm{p}-2$

if $\ell_{\mathrm{p}-1} / \ell_{\mathrm{p}}<\overline{\mathrm{c}}_{\mathrm{p}-1}$ and $\ell_{\mathrm{p}-2} /\left[\left(\ell_{\mathrm{p}}+\ell_{\mathrm{p}-1}\right) / 2\right]>\overline{\mathrm{c}}_{\mathrm{p}-2}$.

Otherwise, go to next step.

Claim that $\overline{\mathrm{q}}=\mathrm{i}$

if $\ell_{\mathrm{p}-1} / \ell_{\mathrm{p}}<\overline{\mathrm{c}}_{\mathrm{p}-1}, \ldots$,

and $\ell_{\mathrm{i}} /\left[\left(\ell_{\mathrm{p}}+\ell_{\mathrm{p}-1}+\ldots+\ell_{\mathrm{i}+1}\right) /(\mathrm{p}-\mathrm{i})\right]>\overline{\mathrm{c}}_{\mathrm{i}}$. Otherwise, go

to next step.

Claim that $\overline{\mathrm{q}}=0$

if $\ell_{\mathrm{p}-1} / \ell_{\mathrm{p}}<\overline{\mathrm{c}}_{\mathrm{p}-1}, \ldots$

and $\ell_{1} /\left[\left(\ell_{\mathrm{p}}+\ell_{\mathrm{p}-1}+\ldots+\ell_{2}\right) /(\mathrm{p}-1)\right]<\overline{\mathrm{c}}_{1}$.

Theorem 2.1: Under the general configuration of eigenvalues of the matrix $\Sigma$ when $\mathrm{q}$ signals are present: $\lambda_{1} \geq \lambda_{2} \geq \ldots \geq \lambda_{\mathrm{q}}>\lambda_{\mathrm{q}+1}=\lambda_{\mathrm{q}+2}=\ldots=\lambda_{\mathrm{p}}, \lambda_{\mathrm{q}} / \lambda_{\mathrm{q}+1} \geq \delta^{*}$, e have

(a) $\mathrm{P}(\mathrm{CE} \mid \underline{\mathrm{R}})$, the probability that the estimate obtained from Procedure $\underline{\mathrm{R}}$ is a lower limit for the number of signals is at least $\left(1+\mathrm{P}^{*}\right) / 2$;

(b) $\mathrm{P}(\overline{\mathrm{CE}} \mid \overline{\mathrm{R}})$, the probability that the estimate obtained from Procedure $\overline{\mathrm{R}}$ is an upper limit for the number of signals is at least $\left(1+\mathrm{P}^{*}\right) / 2$; and

(c) the interval $[\underline{q}, \overline{\mathrm{q}}]$ obtained from Procedure $\underline{\mathrm{R}}$ and $\overline{\mathrm{R}}$ satisfies the probability requirement $\mathrm{P}(\mathrm{q} \in[\underline{\mathrm{q}}, \overline{\mathrm{q}}]) \geq \mathrm{P}^{*}$.

\section{ILLUSTRATED EXAMPLES}

In this section, we give examples to illustrate the estimates of $\overline{\mathrm{c}}_{\mathrm{i}}$ and $\underline{\mathrm{c}}_{\mathrm{i}}$, our procedure parameters. The first example shows how to estimate the procedure parameters when $\mathrm{p}=$ $10, \delta *=4, \mathrm{n}=100$. The second example shows the procedure parameters when $n$ is increased to $n=2000$. Then we use the asymptotic result in Theorems 2.4 to compute the large sample procedure parameters and compare them to the ones obtained from the simulation.

Example 1: We consider the case: $\mathrm{p}=10, \mathrm{n}=100, \delta^{*}=4$. We use simulation to find the $5^{\text {th }}$ and $95^{\text {th }}$ percentiles of the ratios of eigenvalues, $\ell_{\mathrm{i}} /\left[\left(\ell_{\mathrm{p}}+\ldots+\ell_{\mathrm{i}+1}\right) /(\mathrm{p}-\mathrm{i})\right]$ needed in our procedure. All the simulations in this paper use 10000 repetitions. 
The table below shows the $5^{\text {th }}$ and $95^{\text {th }}$ percentiles of $\ell_{\mathrm{i}} /\left[\left(\ell_{\mathrm{p}}+\ldots+\ell_{\mathrm{i}+1}\right) /(\mathrm{p}-\mathrm{i})\right], \mathrm{i}=1, \ldots, \mathrm{p}-1$ when the true number of signals is $i$ where $i \in\{1, \ldots, p-1\}$.

Table 1: $5^{\text {th }}$ and $95^{\text {th }}$ percentiles of $\ell_{\mathrm{i}} /\left[\left(\ell_{\mathrm{p}}+\ldots+\ell_{\mathrm{i}+1}\right) /(\mathrm{p}-\mathrm{i})\right], \mathrm{n}=100$

$\begin{array}{llllllllll}\mathrm{i} & 1 & 2 & 3 & 4 & 5 & 6 & 7 & 8 & 9\end{array}$

$5^{\text {th }}$

percentile $\begin{array}{lllllllll}3.26 & 2.95 & 2.74 & 2.60 & 2.43 & 2.32 & 2.21 & 2.08 & 1.88\end{array}$ $95^{\text {th }}$

$\begin{array}{llllllllll}\text { percentile } & 5.22 & 4.54 & 4.18 & 3.89 & 3.71 & 3.57 & 3.45 & 3.39 & 3.36\end{array}$

Therefore our procedure parameters are $\left(\underline{\mathrm{c}}_{1}, \ldots, \underline{\mathrm{c}}_{9}\right)=($ $5.221,4.541,4.182,4,4,4,4,4,4)$ and $\left(\overline{\mathrm{c}}_{1}, \ldots, \overline{\mathrm{c}}_{9}\right)=(3.26$, $2.95,2.74,2.60,2.43,2.32,2.21,2.08,1.88)$.

Example 2: We increase the sample size $n$ to $n=2000$. The $5^{\text {th }}$ and $95^{\text {th }}$ percentiles of the ratios of eigenvalues, $\ell_{\mathrm{i}} /\left[\left(\ell_{\mathrm{p}}+\ldots+\ell_{\mathrm{i}+1}\right) /(\mathrm{p}-\mathrm{i})\right]$, from simulation are presented in Table 2.

Table 2: $5^{\text {th }}$ and $95^{\text {th }}$ percentiles of $\ell_{\mathrm{i}} /\left[\left(\ell_{\mathrm{p}}+\ldots+\ell_{\mathrm{i}+1}\right) /(\mathrm{p}-\mathrm{i})\right], \mathrm{n}=2000$

$\begin{array}{llllllllll}\mathrm{i} & 1 & 2 & 3 & 4 & 5 & 6 & 7 & 8 & 9\end{array}$

$5^{\text {th }}$

percentile $\begin{array}{lllllllll}3.79 & 3.71 & 3.65 & 3.61 & 3.56 & 3.52 & 3.48 & 3.44 & 3.37\end{array}$

$95^{\text {th }}$

$\begin{array}{llllllllll}\text { percentile } & 4.23 & 4.08 & 4.01 & 3.94 & 3.89 & 3.85 & 3.82 & 3.80 & 3.81\end{array}$

Therefore our procedure parameters are $\left(\underline{\mathrm{c}}_{1}, \ldots, \underline{\mathrm{c}}_{9}\right)=$ $(4.235,4.087,4.005,4,4,4,4,4,4)$ and $\left(\overline{\mathrm{c}}_{1}, \ldots, \overline{\mathrm{c}}_{9}\right)=$ $(3.792,3.714,3.653,3.611,3.562,3.525,3.483,3.440$, $3.371)$.

\section{REFERENCES}

[1] Schmidt, R. O. (1979) "Multiple emitter location and signal parameter estimation", Proceedings of the RADC Spectrum Estimation Workshop, Rome, NY, 243-258.

[2] Wax, M. and Kailath, T. (1985). "Detection of signals by information theoretic criteria", IEEE Transactions on Acoustics, Speech, and Signal Processing, vol. ASSP-33, no. 2, 387-392.

[3] Wax, M, Shan, T., and Kailath, T. (1984) "Spatiotemporal spectral analysis by eigenstructure Methods", IEEE Transactions on Acoustics, Speech, and Signal Processing, vol. ASSP-32, no. 4, 817-828.

[4] Zhao, L. C., P. R. Krishnaiah, and Bai, Z. D. (1986) “On detection of the number of signals in presence of white noise", Journal of Multivariate Analysis, 20, 1, 1-25.

[5] Chen, P. (2002) "A selection procedure for estimating the number of signal components", Journal of Statistical Planning and Inference, 105, 299-311.
[6] Chen, P, Melvin, W. L., and Wicks, M. C. (1999)

"Screening among multivariate normal data", Journal of Multivariate Analysis, 69, 10-29.

[7] Gupta, S. S. and Panchapakesan, S. (1979). Multiple Decision Procedures, Wiley, New York.

[8] Gupta, S. S. and Panchapakesan, S. (1993). "Selection and Screening Procedures in Multivariate Analysis", in Multivariate Analysis: Future Directions (Editor: C. R. Rao), Elsevier Science Publishers, 233-262.

[9] Chen, P. and Wicks, M. C. (2003) "Development of a lower confidence limit for the number of signals", IEEE Transactions on Signal Processing, Vol. 51, No. 6, 14491556. 